\title{
Existence of an Equilibrium for Lower Semicontinuous Information Acquisition Functions
}

\author{
Agnès Bialecki, ${ }^{1}$ Eléonore Haguet, ${ }^{2}$ and Gabriel Turinici ${ }^{3}$ \\ ${ }^{1}$ ENS de Lyon, 15 Parvis René Descartes, BP 7000, 69342 Lyon Cedex 07, France \\ ${ }^{2}$ ENSAE, 3 Avenue Pierre Larousse, 92245 Malakoff Cedex, France \\ ${ }^{3}$ CEREMADE, Université Paris Dauphine, Place du Marechal de Lattre de Tassigny, 75016 Paris, France
}

Correspondence should be addressed to Gabriel Turinici; gabriel.turinici@dauphine.fr

Received 24 July 2013; Accepted 13 March 2014; Published 22 April 2014

Academic Editor: Takashi Matsuhisa

Copyright (C) 2014 Agnès Bialecki et al. This is an open access article distributed under the Creative Commons Attribution License, which permits unrestricted use, distribution, and reproduction in any medium, provided the original work is properly cited.

\begin{abstract}
We consider a two-period model in which a continuum of agents trade in a context of costly information acquisition and systematic heterogeneous expectations biases. Because of systematic biases agents are supposed not to learn from others' decisions. In a previous work under somehow strong technical assumptions a market equilibrium was proved to exist and the supply and demand functions were proved to be strictly monotonic with respect to the price. Here we extend these results under very weak technical assumptions. We also prove that the equilibrium price maximizes the trading volume and further additional properties (such as the antimonotonicity of the trading volume with respect to the marginal information price).
\end{abstract}

\section{Introduction}

We consider a continuum of agents that act in a two-period $(t \in\{0, T\})$ market consisting of a single asset of value $V$. The value $V$ is constant and deterministic but unknown to the agents. Each agent constructs an estimation for $V$ in the form of a normal random variable with known mean and variance. The numerical value of the mean, which is not necessarily $V$ and as such can be interpreted as a systematic bias, is given by the estimation method and cannot be changed. However, the variance can be reduced at time $t=0$ by paying a cost, which is a known deterministic function of the variance to be attained. Each agent uses a CARA utility function and constructs the function mapping each triplet (consisting of the market price, the estimation mean, and the estimation variance) to the optimal number of units to trade. The sum of all such functions from all agents results at time $t=0$ in aggregate market demand and supply functions; the price of the asset is chosen to clear the market (we prove in particular that such a price exists and is unique). This price can be different from the real value $V$ and in practice it will. The agents close their position at final time $t=T$. This paper investigates the following questions: existence of an equilibrium, continuity of supply and demand functions, and interpretation of the equilibrium price as the value maximizing the liquidity (trading volume).

The paper is organized as follows. The rest of this section presents a literature overview. In Section 2 the model is explained and the fundamental Assumption 3 is introduced. In Sections 3 and 3.1, we prove the existence of an equilibrium and important properties of the liquidity (here defined as the transaction volume); in particular we prove that the equilibrium price maximizes the trading volume. We apply our results to a Grossmann-Stiglitz framework in Section 4. Finally, in Section 5 we show that the liquidity is inversely correlated with the marginal price of information.

1.1. Literature Overview. The model has two important ingredients:

(i) the existence of heterogeneous beliefs (or expectations) biases among a continuum of agents;

(ii) the fact that the information is costly (the literature refers to "information acquisition" cost).

There are many models that explain how disagreements between agent estimations generate investment decisions and trading volume. The importance of the heterogeneity of 
opinions on the future value of a financial instrument and its use in speculation has been recognized as early as Keynes (see [1]) who invokes the "beauty contest" metaphor to explain how speculators infer the future (consensus) price.

A model of speculative trading in a large economy with a continuum of agents with heterogeneous beliefs was presented in $[2,3]$ (see also the references within). They demonstrate the existence of price amplification effects and show that the equilibrium prices can be different from the rational expectation equilibrium price. It is also shown that trading volume is positively related to the directions of price changes and they explain the recurrent presence of diverse beliefs. We also refer to [4] and references within for a survey on how heterogeneous beliefs among agents generate speculation and trading.

The difference-of-opinion approach (see $[5,6])$ does not consider "noise agents" but on the contrary obtains diverse posterior beliefs from the differences in the way agents interpret common information. They focus on the implications of the dispersion in beliefs on the price level or direction. Yet another different method explains diverse posterior beliefs by relaxing the assumption of a common prior distribution (see [7]); the authors also model the learning process which enables a convergence towards a common estimation when more information is available. Such a framework was invoked for modeling asset pricing during initial public offerings, but not for other speculative circumstances. Finally, Pagano [8] analyze the implications of low liquidity in a market and propose appropriate incentive schemes to shift the market to an equilibrium characterized by a higher number of transactions.

An important advance has been to recognize that the dynamics of the information gathering is important; it was thus established how the presence of private information and noise (liquidity) agents interact with market price and volume (see, e.g., [9-11], for recent related endeavors). More specifically it was recognized (the so called "GrossmanStiglitz paradox") that it is not always optimal for the agents to obtain all the information on a particular asset. This remark is of importance in our paper in the following because, as explained in Section 2, our model allows each agent to choose his level of precision related to the estimation of the true value of the traded asset. In the classical paper of [12] and in subsequent related works [13-18] a framework is proposed where the information is costly and agents can pay more to lower their uncertainty on the future value of the risky asset. Verrechia derives a closed form solution which requires some particular assumptions. These include the convexity of the cost function with respect to the precision (the precision being the inverse of the estimate's variance). On the contrary our cost function is here only lower semicontinuous. Our approach also differs in a more fundamental way in that we suppose that heterogeneity of estimations is given but arbitrary, that is, not centered around the correct price. Moreover, the Verrecchia model relies on the heterogeneity of risk tolerances in the CARA utility function while in our work the price formation mechanism does not require such an assumption, the heterogeneity in estimations being enough. Also, in this model, the endowments of the agents do not play any role and in particular are not required to obtain an equilibrium. The paper extends a previous work [19] where stronger technical assumptions were invoked.

\section{The Model}

We consider a two-period model, $t=0$ and $t=T$, in which a risky security of value $V$ is traded. The value $V$ is unknown to the agents and each participant $x$ in the market constructs an estimate $\widetilde{A}^{x}$ for $V$ at $t=0, \widetilde{A}^{x}$ being a random variable. For simplicity, we suppose that $\widetilde{A}^{x}$ has a normal distribution and that $\widetilde{A}^{x_{1}}$ and $\widetilde{A}^{x_{2}}$ are independent if $x_{1}$ and $x_{2}$ are two distinct agents (this independence assumption is motivated by the existence of an individual bias for each agent as explained below). Also, we assume that the mean and the variance of $\widetilde{A}^{x}$ are, respectively, given by $A^{x}$ and $\left(\sigma^{x}\right)^{2}$, both mean and variance being known to the agent $x$. As in [12] we work with the precision $B^{x}=1 /\left(\sigma^{x}\right)^{2}$ instead of the variance $\left(\sigma^{x}\right)^{2}$.

Many estimation procedures can output results in the form of a normal variable with known mean and variance, the most known example being a Kalman-Bucy filter; see [20] for details.

Note that we do not model here the riskless security, but everything works as if the numeraire was the riskless security; from a technical point of view this allows setting the interest rate to zero.

An important remark is that each agent has his own bias attached to the estimate $\widetilde{A}^{x}$ because he has his own procedure to interpret the available information. It may be due to personal optimism or pessimism (e.g., the agent is a "bull" or "bear") or may be correlated with some exogenous factors, such as overall economic outlooks, commodities evolution, and geopolitical factors, which each agent interprets with a specific systematic bias. See also the cited references for additional discussion on how agents interpret the information they obtain. We assume that the bias $A^{x}-V$ of agent $x$ does not depend on the precision $B^{x}$ to be attained and only depends on the agent; the value $A^{x}$ associated to an agent is known only by him. The agent does not influence $A^{x}$ in any way during the process of forecasting; his forecasting process is not influenced by other agents' decisions; that is, there is no collective learning in this model. Hence, two different agents $x_{1}$ and $x_{2}$ have generically different biases $A^{x_{1}}-V$ and $A^{x_{2}}-V$ and thus different estimation averages $A^{x_{1}}$ and $A^{x_{2}}$. This is not a collateral property of the model. It is instead the mere reason for which the agents trade. They trade because they have different (heterogeneous) expectations on the final value of the security.

We define $\rho(A)$ to be the distribution of $A^{x}$ among the agents; neither the law of the distribution $\rho(A)$ nor any moments or statistics is known by the agents. We also introduce the expected value with respect to $\rho(\cdot)$, which is denoted by $\mathbb{E}^{A}$; see also [21] for related works on empirical estimation of such a distribution $\rho$. We do not assume the law of $\rho$ to be normal or have particular properties (except technical Assumption 9).

From a theoretical point of view, it is interesting to explore the case when $\mathbb{E}^{A}(A)=V$. This means that the average 
estimate is $V$, so that the agents are neither overpricing nor underpricing the security with respect to its (unknown) value. However, we will see that this does not necessarily indicate that the market price is $V$.

The only parameter the agent can control is the accuracy of the result, that is, the precision $B^{x}$. However, this has a cost: the agent has to pay $f\left(B^{x}\right)$ to obtain the precision $B^{x}$. The precision cost function $f: \mathbb{R}_{+} \rightarrow \mathbb{R}_{+}$is defined on positive numbers but if needed we set by convention $f(b)=\infty$ for any $b<0$. See also [22] for an example involving a power function and [18] for a structural model to motivate such a function.

Such a model is relevant in the case of high expense for information sources, for instance, news broadcasting fees. The expense also involves the reward of research personnel or the need for more accurate computer simulations.

Based on his estimations the agent $x$ decides at time $t=0$ to trade a quantity of $\theta^{x}$ security units. When $\theta^{x}$ is positive, the agent is long, so he buys the security, whereas when $\theta^{x}$ is negative, he is short; he sells it.

Hence, each agent is characterized by three parameters: his mean estimate $A^{x}$, the precision $B^{x}$ of the estimate (that comes at a cost $f\left(B^{x}\right)$ ), and the quantity of traded units, $\theta^{x}$.

The agents buy or sell the security at time $t=0$ by formulating demand and supply functions depending on the price. The market price at time $t=0$ is chosen to clear the aggregate total demand/supply.

Remark 1. The price that clears the market is also called market equilibrium price. Note however that the uniqueness of the equilibrium is, at this stage, not proved.

We set the investment horizon of all agents to be the final time $t=T$ which is the time when each agent liquidates his initial position. Each agent supposes that this final transaction takes place at a price in agreement with his initial estimation.

In order to describe the model for the market price, we introduce for any price $p>0$ the basic notions of total supply $S(p)$ and total demand $D(p)$ defined as

$$
D(p)=\mathbb{E}^{A}\left(\theta_{+}\right), \quad S(p)=\mathbb{E}^{A}\left(\theta_{-}\right),
$$

where for any real number $a$ we define $a_{+}=\max \{a, 0\}$, $a_{-}=\max \{-a, 0\}$.

A price $p^{*}$ such that $S\left(p^{*}\right)=D\left(p^{*}\right)$ is said to clear the market. From the definition of $D(\cdot)$ and $S(\cdot)$ in (1) this is equivalent to saying that $\mathbb{E}^{A}(\theta)=0$; that is, at the price $p^{*}$, the overall (signed) demand is zero. Note that such a price may not exist or may not be unique. Hence, one of the goals of the paper is to prove existence and uniqueness of $p^{*}$.

The transaction volume at some price $p$ is the number of units that can be exchanged at that price and is defined as follows:

$$
\operatorname{TV}(p)=\min \{S(p), D(p)\}
$$

A price $p^{*}$ for which $\operatorname{TV}(\cdot)$ reaches its maximum is of particular interest because it maximizes the total number of asset units being exchanged. Note that such a price may not exist and may also be nonunique.

Let us recall the following result (see [19] for the proof).

Theorem 2. Suppose that functions $S(p), D(p)$ are continuous and positive, $S(0)=0$, and $\lim _{p \rightarrow \infty} D(p)=0$. Consider the following.

(A) If $S(p)$ is increasing, not identically zero, and $D(p)$ is decreasing, then there exists at least one price $p^{*}<\infty$ such that $S\left(p^{*}\right)=D\left(p^{*}\right)$; moreover $T V\left(p^{*}\right) \geq T V(p)$ for all $p \geq 0$.

(B) In addition to previous assumptions suppose that $S(p)$ is strictly increasing and $\lim _{p \rightarrow \infty} S(p)>0$, whereas $D(p)$ is strictly decreasing and such that $D(0)>0$. Then the following statements are true.

(1) There exists a unique $p_{1}^{*}$ such that $S\left(p_{1}^{*}\right)=$ $D\left(p_{1}^{*}\right)$.

(2) There exists a unique $p_{2}^{*}$ such that $T V\left(p_{2}^{*}\right) \geq$ $T V(p)$ for all $p \geq 0$.

(3) Moreover $p_{1}^{*}=p_{2}^{*}$.

Recall that $F: \mathbb{R}_{+} \rightarrow \mathbb{R}_{+} \cup\{+\infty\}$ is said to be lower semicontinuous (denoted by "l.s.c.") if for any $x \in \mathbb{R}_{+}$

$$
F(x) \leq \liminf _{y \rightarrow x} F(y) .
$$

A function $G$ such that $-G$ is l.s.c. is said to be upper semicontinuous (denoted by "u.s.c.").

For any function $\zeta: \mathbb{R}_{+} \rightarrow \mathbb{R}_{+} \cup\{+\infty\}$ we define

$$
\underline{\zeta(x)}=\liminf _{y \rightarrow x} \zeta(y), \quad \underline{\zeta^{\prime}(x)}=\liminf _{y \rightarrow x} \frac{\zeta(y)-\zeta(x)}{y-x} .
$$

In particular $f^{\prime}(0)=\liminf _{y \rightarrow 0}((f(y)-f(0)) / y)$. Denote by $\left(f^{\prime}(0)\right)$ its positive part.

Let us introduce an important assumption of this paper.

Assumption 3. We say that a function $f: \mathbb{R}_{+} \rightarrow \mathbb{R}_{+} \cup$ $\{+\infty\}$ satisfies Assumption 3 if $f(0)<\infty, f$ is lower semicontinuous, and there exists $\overline{\beta>0}$ such that

$$
\liminf _{x \rightarrow \infty} \frac{f(x)}{x^{1+\beta}}>0 .
$$

Remark 4 . The quantity $f(0)<\infty$ represents the residual cost, when precision approaches zero, to enter the market. It is not related to the precision (because there is none in the limit) but to the fixed costs to trade on the market (independent of the quantity). A market with infinite fixed costs is not realistic.

The assumption $f(0)<\infty$ implies, by lower semicontinuity, that $f(0)<\infty$ and is realistic in that it demands that the price of zero precision be finite.

In order to model the choices of the agents, we consider that the agents maximize a CARA-type expected utility 
function (see [23]); that is, if the output is the random variable $X$, they maximize $\mathbb{E}\left(-e^{-\lambda X}\right)$. Note that if $X$ is a normal random variable with mean $\mathbb{E}(X)$ and variance $\operatorname{var}(X)$, then maximizing $\mathbb{E}\left(-e^{-\lambda X}\right)$ is equivalent to maximizing the meanvariance utility function $\mathbb{E}(X)-((\lambda / 2) \operatorname{var}(X))$. We refer to (6) for the treatment of degenerate normal variables with infinite variance. The parameter $\lambda \in \mathbb{R}_{+}$is called the risk aversion coefficient. Note that all agents have here the same utility function; see for instance $[24,25]$ who argue that differences in preferences are not a major factor in explaining the magnitude of trade in speculative markets.

Of course, the expected wealth of the agent at time $t=T$ is a function of $\theta^{x}$ and $B^{x}$. It is computed under the assumption that each agent enters the transaction (buys or sells) at time $t=0$ at the market price and exits the transaction (sells or buys) at time $t=T$ at a price coherent with his estimation; that is, we condition on the available information at time $t=$ 0 . Thus, for a given price $p$, which is not necessarily equal to the market equilibrium price $\overline{\mathscr{P}}$, the average expected wealth at time $t=T$ of the agent $x$ denoted by $u^{x}$ is given by $u^{x}=$ $\theta^{x}\left(A^{x}-p\right)-f\left(B^{x}\right)$. The variance of the wealth, denoted by $v^{x}$, is given by $v^{x}=\left(\theta^{x}\right)^{2} / B^{x}$.

Thus, for a given price $p$ (not necessarily the market equilibrium price $\overline{\mathscr{P}}$ ) the fact that agent $x$ optimizes his CARA utility function is equivalent to saying that he optimizes with respect to $\theta^{x}$ and $B^{x}$ his mean-variance utility:

$$
J\left(\theta^{x}, B^{x}\right)= \begin{cases}\theta^{x}\left(A^{x}-p\right)-f\left(B^{x}\right)-\frac{\lambda}{2} \frac{\left(\theta^{x}\right)^{2}}{B^{x}} & \text { if } B^{x}, \theta^{x}>0 \\ -\infty & \text { if } B^{x}=0, \theta^{x}>0 \\ -f(0) & \text { if } B^{x}=\theta^{x}=0 .\end{cases}
$$

\section{Existence of the Transaction Volume}

Each agent $x$ is characterized by his own bias $A^{x}$. The agents consider the market price as being fixed, which means they cannot influence it directly. They do not know any statistics on $\rho$ so the market price is not directly informative, but the acquired information is. Therefore, their strategy depend on two values: the bias $A$ and the market price $p$.

Under Assumption 3, the agent chooses the optimal pair of precision $B_{\text {opt }}(p, A ; f)$ and demand/supply $\theta_{\text {opt }}(p, A ; f)$, that is, the value of the pair maximizing the following expression:

$$
\mathscr{J}(y, z)= \begin{cases}y(A-p)-f(z)-\frac{\lambda}{2} \frac{y^{2}}{z} & \text { if } y, z>0 \\ -\infty & \text { if } z=0, y>0 \\ -f(0) & \text { if } y=z=0\end{cases}
$$

so that

$$
\mathscr{J}\left(\theta_{\text {opt }}(p, A ; f), B_{\text {opt }}(p, A ; f)\right) \geq \mathscr{J}(y, z), \quad \forall y, z \geq 0 .
$$

Let $g_{p, A ; f}(X)=\left((p-A)^{2} / 2 \lambda\right) X-f(X)$ and let $\alpha$ be the function defined by $\alpha(p, A)=(p-A)^{2} / 2 \lambda$. To simplify the notations we sometimes write only $g_{p, A}, g_{p}$, or $g$ instead of $g_{p, A ; f}$ and $\theta_{\text {opt }}(p, A) / B_{\text {opt }}(p, A)$ instead of $\theta_{\text {opt }}(p, A ; f) / B_{\text {opt }}(p, A ; f)$; likewise $\alpha$ stands for $\alpha(p, A)$.

Lemma 5. Under Assumption 3, for any $p$ and $A$, there exists a pair $\left(B_{o p t}(p, A), \theta_{\text {opt }}(p, A)\right)$ such that $(8)$ is satisfied.

Proof. Since $f$ satisfies Assumption 3, there exists $x_{1}>0$ and some positive constant $C_{1}$ such that $f(x) \geq C_{1} x^{1+\beta}$ for all $x \geq x_{1}$. In particular for

$$
x>\max \left\{x_{1},\left(\frac{2 \alpha}{C_{1}}\right)^{1 / \beta},\left(\frac{2 f(0)}{C_{1}}\right)^{1 /(1+\beta)}\right\},
$$

we have $g(x)<-f(0)=g(0)$. Since $f$ is l.s.c. then $g$ is u.s.c.; it follows that $g$ attains its maximum on $\mathbb{R}_{+}$in the interval $\left[0, \max \left\{x_{1},\left(2 \alpha / C_{1}\right)^{1 / \beta},\left(2 f(0) / C_{1}\right)^{1 /(1+\beta)}\right\}\right]$. We set $B_{\text {opt }}(p, A)$ to be one such maximum (it may not be unique) and set $\theta_{\text {opt }}(p, A)=(A-p) B_{\text {opt }}(p, A) / \lambda$.

Note that $B_{\text {opt }}(p, A)=0$ implies $\theta_{\text {opt }}(p, A)=0$; thus

$$
\forall y>0: \mathscr{J}\left(\theta_{\text {opt }}(p, A), B_{\text {opt }}(p, A)\right)>-\infty=\mathscr{J}(y, 0) \text {. }
$$

When $y=z=0$, one has

$$
\begin{aligned}
\mathscr{J}(0,0) & =g(0) \leq g\left(B_{\mathrm{opt}}(p, A)\right) \\
& =\mathscr{J}\left(\theta_{\mathrm{opt}}(p, A), B_{\mathrm{opt}}(p, A)\right) .
\end{aligned}
$$

Let $y, z>0$. Since $\mathscr{J}$ is a parabola with negative leading coefficient with respect to its first argument, it follows that

$$
\begin{aligned}
\mathscr{J}(y, z) & \leq \mathscr{J}\left(\frac{(A-p) z}{\lambda}, z\right)=g(z) \leq g\left(B_{\mathrm{opt}}(p, A)\right) \\
& =\mathscr{J}\left(\theta_{\mathrm{opt}}(p, A), B_{\mathrm{opt}}(p, A)\right) .
\end{aligned}
$$

Remark 6. Note that the formula $\theta_{\text {opt }}(p, A)=(A-$ $p) B_{\text {opt }}(p, A) / \lambda$ is completely compatible with previous works, see [26] p575, although here we have no assumption on budget constraints and the risk-less interest rate is set to zero.

In order to prove the existence of an equilibrium we need the following auxiliary results (Lemmas 7-11).

Lemma 7. Under Assumption 3, let $\left(p_{1}, A_{1}\right),\left(p_{2}, A_{2}\right)$ be such that $\alpha_{1} \leq \alpha_{2}$, where $\alpha_{k}=\alpha\left(p_{k}, A_{k}\right)$. Then $B_{\text {opt }}\left(p_{1}, A_{1}\right) \leq$ $B_{\text {opt }}\left(p_{2}, A_{2}\right)$. One says that $B_{\text {opt }}(p, A)$ is increasing with respect to $\alpha$. In particular, for fixed $A$, one has the following:

(i) $B_{o p t}(p, A)$ is increasing with respect to $p$ on the interval ]A, $\infty[$

(ii) $B_{o p t}(p, A)$ is decreasing with respect to $p$ on the interval ]0, $A[$. 
Proof. Let $B_{k}=B_{\text {opt }}\left(p_{k}, A_{k}\right)$, for $k=1,2$. Recall that $B_{k}$ optimizes $\alpha_{k} B-f(B)$ with respect to $B$. Then

$$
\begin{aligned}
\alpha_{1} B_{1}-f\left(B_{1}\right) & \geq \alpha_{1} B_{2}-f\left(B_{2}\right) \\
& =\alpha_{2} B_{2}-f\left(B_{2}\right)+\left(\alpha_{1}-\alpha_{2}\right) B_{2} \\
& \geq \alpha_{2} B_{1}-f\left(B_{1}\right)+\left(\alpha_{1}-\alpha_{2}\right) B_{2} .
\end{aligned}
$$

Thus, $\alpha_{1} B_{1} \geq \alpha_{2} B_{1}+\left(\alpha_{1}-\alpha_{2}\right) B_{2}$ and hence $\left(\alpha_{1}-\alpha_{2}\right)\left(B_{1}-B_{2}\right) \geq$ 0 , which gives the conclusion.

Lemma 8. Under Assumption 3, let $\alpha_{n}=\alpha\left(p_{n}, A_{n}\right), n \geq 0$, be a sequence such that $\alpha_{n} \rightarrow{ }_{n \rightarrow+\infty} \alpha_{0}$ but $B_{o p t}\left(p_{n}, A_{n}\right)$ does not converge to $B_{o p t}\left(p_{0}, A_{0}\right)$. The set of such $\alpha_{0}$ is at most countable. In particular, if $p$ is fixed, then the set of $A$ such that $B_{o p t}(p, A)$ is discontinuous with respect to $A$ is countable. An analogous result holds if $A$ is fixed.

Proof. Let $B_{n}=B_{\text {opt }}\left(p_{n}, A_{n}\right)$, for $n \geq 0$. Without loss of generality, we only investigate the case when the sequence $\alpha_{n}$ converges decreasingly to $\alpha_{0}$. Then, we have $B_{n} \geq B_{0}, \forall n \geq 0$.

Since $B_{n}$ does not converge to $B_{0}$, let $\eta=\left(\lim _{n \rightarrow+\infty} B_{n}\right)-$ $B_{0}$. Note that $\eta>0$ and $B_{n} \geq B_{0}+\eta, \forall n \geq 0$. Also recall that

$$
\alpha_{n} B_{n}-f\left(B_{n}\right) \geq \alpha_{n} B-f(B), \quad \forall B .
$$

Yet, since $-f$ is u.s.c.,

$$
\alpha_{0}\left(B_{0}+\eta\right)-f\left(B_{0}+\eta\right) \geq \limsup _{n \rightarrow \infty}\left(\alpha_{n} B_{n}-f\left(B_{n}\right)\right),
$$

and for fixed $B, \alpha_{n} B-f(B)$ converges to $\alpha_{0} B-f(B)$. In the limit when $n \rightarrow \infty$, it holds that

$$
\alpha_{0}\left(B_{0}+\eta\right)-f\left(B_{0}+\eta\right) \geq \alpha_{0} B-f(B), \quad \forall B .
$$

This implies that $B_{0}+\eta$ is also a maximum for $\alpha_{0} B-$ $f(B)$. From this we deduce that $g_{\alpha_{0}}$ has at least two distinct maximums, $B_{0}$ and $B_{0}+\eta$.

Let $\alpha$ be such that $g_{\alpha}$ has at least two distinct minimums $x_{\alpha}^{1}$ and $x_{\alpha}^{2}$ with $x_{\alpha}^{1}<x_{\alpha}^{2}$; we associate to $\alpha$ a rational number $q_{\alpha}$ such that $\left.q_{\alpha} \in\right] x_{\alpha}^{1}, x_{\alpha}^{2}$ [. Take $\alpha$ and $\tilde{\alpha}$ such that $\alpha \neq \tilde{\alpha}$; to fix notations suppose $\alpha<\tilde{\alpha}$. Then by the previous result $x_{\alpha}^{2} \leq x_{\tilde{\alpha}}^{1}$; moreover $q_{\alpha}<x_{\alpha}^{2} \leq x_{\tilde{\alpha}}^{1}<q_{\tilde{\alpha}}$; that is, $q_{\alpha} \neq q_{\tilde{\alpha}}$. Thus the set of $\alpha$ such that $g_{\alpha}$ has at least two distinct minimums is of cardinality smaller than the cardinality of $\mathbb{Q}$, that is, at most countable. Since continuity can only fail when $g_{\alpha}$ has nonunique maximum, the conclusion follows.

Assumption 9. We say that $\rho(A)$ satisfies Assumption 9 if $\rho$ is absolutely continuous with respect to the Lebesgue measure and

$$
\int_{0}^{\infty} A^{1+2 / \beta} \rho(A) d A<\infty
$$

Lemma 10. Let $S(f, p)$ and $D(f, p)$ (or in short notation $S(p)$ and $D(p)$ when function $f$ is implicit) be defined by

$$
\begin{aligned}
& S(f, p)=\frac{1}{2 \lambda} \int_{0}^{\infty}(A-p)_{-} B_{o p t}(p, A ; f) \rho(A) d A, \\
& D(f, p)=\frac{1}{2 \lambda} \int_{0}^{\infty}(A-p)_{+} B_{o p t}(p, A ; f) \rho(A) d A .
\end{aligned}
$$

Then under Assumptions 3 and $9 S(p)$ and $D(p)$ are finite, continuous, and monotonic. Moreover $S(0)=0=\lim _{p \rightarrow \infty} D(p)$.

Proof. To prove that $S(p)$ and $D(p)$ are finite we recall that the maximum of $g_{p, A}$ is attained in the interval $\left[0, \max \left\{x_{1},\left(2 \alpha / C_{1}\right)^{1 / \beta},\left(2 f(0) / C_{1}\right)^{1 /(1+\beta)}\right\}\right]$; that is, $B_{\text {opt }}(p, A)$ $\leq \max \left\{x_{1},\left(2 \alpha / C_{1}\right)^{1 / \beta},\left(2 f(0) / C_{1}\right)^{1 /(1+\beta)}\right\}$. Recalling that $\alpha=$ $(A-p)^{2} / 2 \lambda$ it follows that both integrals are bounded (modulo some constant) by $\int_{0}^{\infty} A^{1+2 / \beta} \rho(A) d A$; that is, $S(p)$ and $D(p)$ are finite for all $p \geq 0$.

Let $p_{n}$ be a sequence increasingly converging to $p$. For any $X$, the set of $A$ such that $B_{\text {opt }}(X, A)$ is discontinuous is at most countable. Denote it by $\mathscr{B}_{X}$. Let $\mathscr{B}=\mathscr{B}_{p} \cup\left(\bigcup_{n=1}^{+\infty} \mathscr{B}_{p_{n}}\right) . \mathscr{B}$ is also clearly countable and thus $\rho(\mathscr{B})=0$.

Let $\zeta_{n}(A)=\left(A-p_{n}\right)_{-} B_{\text {opt }}\left(p_{n}, A\right)$ and $\zeta(A)=$ $(A-p)_{-} B_{\text {opt }}(p, A)$. Then $\lim _{n \rightarrow+\infty} \zeta_{n}(A)=\zeta(A)$, for all $A$ with the possible exception of the null set $\mathscr{B}$. Also, the sequence $\zeta_{n}$ is increasing.

Then from the Beppo-Levi theorem, the following holds:

$$
\begin{aligned}
\lim _{n \rightarrow+\infty} S\left(p_{n}\right) & =\lim _{n \rightarrow+\infty} \frac{1}{2 \lambda} \int_{0}^{+\infty}\left(A-p_{n}\right)_{-} B_{\text {opt }}\left(p_{n}, A\right) \rho(A) d A \\
& =\frac{1}{2 \lambda} \int_{0}^{+\infty}(A-p)_{-} B_{\text {opt }}(p, A) \rho(A) d A=S(p) .
\end{aligned}
$$

This proves sequential continuity of $S(p)$ and thus its continuity. The monotonicity is a consequence of the monotonicity of $B_{\text {opt }}(p, A)$. This result also holds for the demand $D(p)$, recalling that $-D(p)$ is increasing and lower-bounded.

The property $S(0)=0$ is trivial. To prove $\lim _{p \rightarrow \infty} D(p)=$ 0 it is sufficient to use the above upper bound for $B_{\text {opt }}(p, A)$ and $\lim _{p \rightarrow \infty} \int_{p}^{\infty} A^{1+2 / \beta} \rho(A) d A=0$.

Recall that $S(p)$ is increasing on $[0,+\infty$ [ but in order to use Theorem 2 we need to prove its strict monotonicity.

Lemma 11. Under Assumptions 3 and 9 and supposing ${\underline{(f}(0))_{+}}_{+}<\infty$ the following hold:

(1) $S(p)$ is strictly increasing on $] \sqrt{2 \lambda\left(\underline{\left.f^{\prime}(0)\right)}+\right.}+$ $\inf (\operatorname{supp}(\rho)),+\infty[$

(2) $S(0)=0$;

(3) $\lim _{p \rightarrow+\infty} S(p)>0$;

(4) $D(p)$ is strictly decreasing on $[0, \sup (\operatorname{supp}(\rho))-$ $\sqrt{2 \lambda\left(\underline{f(0))}_{+}\right]}$;

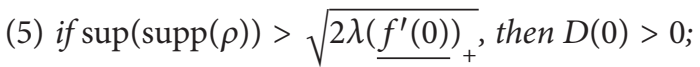

(6) $\lim _{p \rightarrow+\infty} D(p)=0$.

Remark 12. The assumption $\left(\underline{f}^{\prime}(0)\right)_{+}<\infty$ will be relaxed in Section 3.1, cf. Theorem 18. 
Proof. Note that $\left(f^{\prime}(0)\right)_{+}<\infty$ implies in particular continuity of $f(B)$ at $B=0$. Letting $p$ and $p^{\prime}$ be such that $p>p^{\prime}>A \geq 0$,

$$
\begin{aligned}
& S(p)-S\left(p^{\prime}\right)=\frac{1}{2 \lambda} \int_{0}^{\infty}[(A-p)_{-} B_{\mathrm{opt}}(p, A) \\
&\left.-\left(A-p^{\prime}\right)_{-} B_{\mathrm{opt}}\left(p^{\prime}, A\right)\right] \rho(A) d A \\
&=\frac{1}{2 \lambda} \int_{0}^{\infty}\left[(A-p)_{-} B_{\mathrm{opt}}(p, A)\right.\left.-\left(A-p^{\prime}\right)_{-} B_{\mathrm{opt}}(p, A)\right] \rho(A) d A \\
&+\frac{1}{2 \lambda} \int_{0}^{\infty}\left[\left(A-p^{\prime}\right)_{-} B_{\mathrm{opt}}(p, A)\right. \\
&\left.\quad-\left(A-p^{\prime}\right)_{-} B_{\mathrm{opt}}\left(p^{\prime}, A\right)\right] \rho(A) d A .
\end{aligned}
$$

Since $B_{\text {opt }}$ is increasing if $p>A$,

$$
\frac{1}{2 \lambda} \int_{0}^{\infty}\left(A-p^{\prime}\right)_{-}\left(B_{\text {opt }}(p, A)-B_{\text {opt }}\left(p^{\prime}, A\right)\right) \rho(A) d A \geq 0 .
$$

Hence,

$$
\begin{aligned}
& S(p)-S\left(p^{\prime}\right) \\
& \quad \geq \frac{1}{2 \lambda} \int_{0}^{\infty}\left((A-p)_{-}-\left(A-p^{\prime}\right)_{-}\right) B_{\mathrm{opt}}(p, A) \rho(A) d A .
\end{aligned}
$$

Note that $A<p^{\prime}<p$ implies that $\left((A-p)_{-}-\left(A-p^{\prime}\right)_{-}\right)>0$. So, in order to prove the strict inequality in the estimation above, it is sufficient to prove that $B_{\text {opt }}(p, A)>0$ with $A$ in the support of $\rho$. Yet

$$
B_{\text {opt }}(p, A)=\arg \max _{B} g_{p}(B)=\arg \max _{B}(\alpha B-f(B)) .
$$

Therefore we only need to prove that there exists $B$ such that $\alpha B-f(B)>0$ with $A$ in the support of $\rho$. A sufficient condition is that the upper limit of derivative of $\alpha B-f(B)$ at $B=0$ be strictly positive. This means $\alpha-\left(\underline{\left.f^{\prime}(0)\right)}{ }_{+}>0\right.$ which is equivalent to $(p-A)^{2} / 2 \lambda>\left(\underline{\left.f^{\prime}(0)\right)}\right.$. Recalling that $p>A$, the latter condition can be rewritten as $p-A>\sqrt{2 \lambda\left(\underline{f}^{\prime}(0)\right)}$ or else $p>A+\sqrt{2 \lambda\left(f^{\prime}(0)\right)}$, for at least one $A$ in the support of $\rho$. Therefore $S(p)-S\left(p^{\prime}\right)>0$ as soon as $p$ is in ]$\sqrt{2 \lambda\left(f^{\prime}(0)\right)}+\inf (\operatorname{supp}(\rho)),+\infty[$. This implies strict monotonicity for $S(p)$ on $] \sqrt{2 \lambda\left(f^{\prime}(0)\right)}+\inf (\operatorname{supp}(\rho)),+\infty[$ and hence also on the interval $\left[\sqrt{2 \lambda\left(f^{\prime}(0)\right)}+\inf (\operatorname{supp}(\rho)),+\infty[\right.$.

We have already seen that $S(0)=0$. Moreover since the supply is strictly increasing on $\left[\sqrt{2 \lambda\left(f^{\prime}(0)\right)}+\right.$ $\inf (\operatorname{supp}(\rho)),+\infty[$ and increasing on $[0,+\infty[$, it holds that $\lim _{p \rightarrow+\infty} S(p)>0$.
For the monotonicity of the demand, let $p$ and $p^{\prime}$ be such that $A>p>p^{\prime}$. Then

$$
\begin{aligned}
& D(p)-D\left(p^{\prime}\right)=\frac{1}{2 \lambda} \int_{0}^{\infty}\left[(A-p)_{+} B_{\mathrm{opt}}(p, A)\right.\left.-\left(A-p^{\prime}\right)_{+} B_{\mathrm{opt}}\left(p^{\prime}, A\right)\right] \rho(A) d A \\
&=\frac{1}{2 \lambda} \int_{0}^{\infty}\left[(A-p)_{+} B_{\mathrm{opt}}(p, A)\right.\left.-\left(A-p^{\prime}\right)_{+} B_{\mathrm{opt}}(p, A)\right] \rho(A) d A \\
&+\frac{1}{2 \lambda} \int_{0}^{\infty}\left[\left(A-p^{\prime}\right)_{+} B_{\mathrm{opt}}(p, A)\right. \\
&\left.\quad-\left(A-p^{\prime}\right)_{+} B_{\mathrm{opt}}\left(p^{\prime}, A\right)\right] \rho(A) d A .
\end{aligned}
$$

Since $B_{\text {opt }}$ is decreasing for $A>p>p^{\prime}$, we have

$$
\frac{1}{2 \lambda} \int_{0}^{\infty}\left(A-p^{\prime}\right)_{+}\left(B_{\mathrm{opt}}(p, A)-B_{\mathrm{opt}}\left(p^{\prime}, A\right)\right) \rho(A) d A \leq 0 \text {. }
$$

Hence,

$$
\begin{aligned}
D(p)-D\left(p^{\prime}\right) \leq \frac{1}{2 \lambda} \int_{0}^{\infty} & \left((A-p)_{+}-\left(A-p^{\prime}\right)_{+}\right) \\
& \times B_{\mathrm{opt}}(p, A) \rho(A) d A .
\end{aligned}
$$

Note that $A>p>p^{\prime}$ implies that $(A-p)_{+}-\left(A-p^{\prime}\right)_{+}<0$. For strict inequality it is sufficient to prove that $B_{\text {opt }}(p, A)>$ 0 . Using the same arguments as in Lemma 11, we have strict monotonicity as soon as $(p-A)^{2} / 2 \lambda>\left(f^{\prime}(0)\right)$.

Recalling that $p<A$, the latter condition can be written as $A-p>\sqrt{2 \lambda\left(f^{\prime}(0)\right)}+$ or else $p<A-\sqrt{2 \lambda\left(f^{\prime}(0)\right)}+$ for at least one $A$ in the support of $\rho$. Therefore, $D(p)-D\left(p^{\prime}\right)<0$ as soon as $p$ is in $] 0, \sup (\operatorname{supp}(\rho))-\sqrt{2 \lambda\left(f^{\prime}(0)\right)}+$ [. This yields strict monotonicity of $D(p)$ on $] 0, \sup (\operatorname{supp}(\rho))-\sqrt{2 \lambda\left(f^{\prime}(0)\right)}[$. Monotonicity also holds on $\left[0, \sup (\operatorname{supp}(\rho))-\sqrt{2 \lambda\left(f^{\prime}(0)\right)_{+}}\right]$ by continuity.

Since $\sup (\operatorname{supp}(\rho))-\sqrt{2 \lambda\left(f^{\prime}(0)\right)}+>0$, we have $B_{\text {opt }}(0, A)>0$ so $D(0)>0$.

Hence, demand is strictly decreasing. Previously we also proved that $\lim _{p \rightarrow+\infty} D(p)=0$.

The above results can be summarized in the following.

Theorem 13. Under Assumptions 3 and 9 and supposing $\left(f^{\prime}(0)\right)+\infty$ the following hold:

(A) there exists at least a $p^{*} \geq 0$ such that $T V\left(p^{*}\right) \geq$ $T V(p), \forall p \geq 0$; moreover $D\left(p^{*}\right)=S\left(p^{*}\right)$;

(B) suppose that $\operatorname{diam}(\operatorname{supp}(\rho))>2 \sqrt{2 \lambda\left(f^{\prime}(0)\right)_{+}}$; then

(1) the functions $B_{\text {opt }}$ and $\theta_{\text {opt }}$ are well defined, 


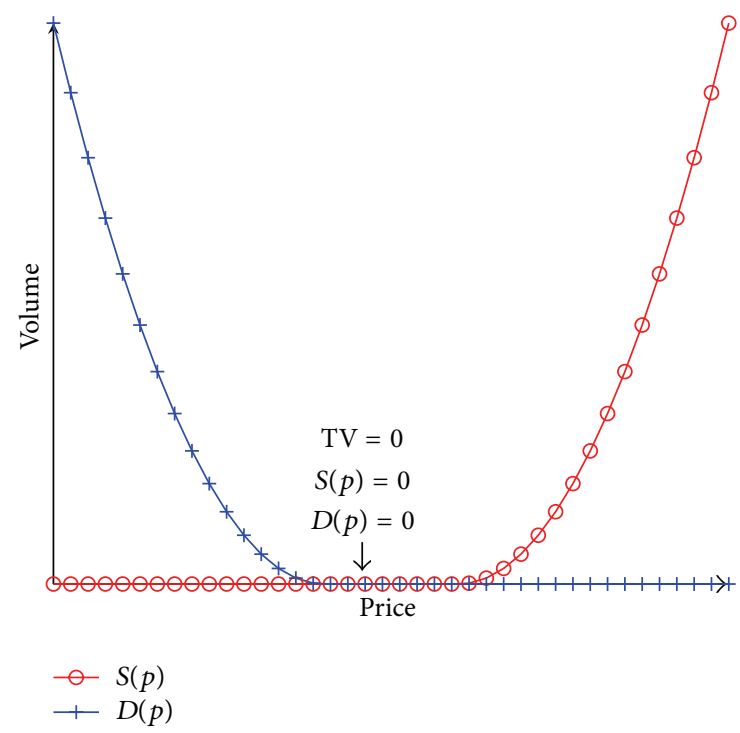

FIGURE 1: Illustration of Remark 14.

(2) there exists a unique $p^{*}>0$ such that $T V\left(p^{*}\right) \geq$ $T V(p), \forall p \geq 0$. Moreover $p^{*}$ is the unique solution of the equation $D\left(p^{*}\right)=S\left(p^{*}\right)$.

Note that the results of [19] are a special case of this Theorem (any convex $C^{2}$ function is l.s.c.).

$\operatorname{Remark} 14$. If $\operatorname{diam}(\operatorname{supp}(\rho)) \leq 2 \sqrt{2 \lambda\left(f^{\prime}(0)\right)}+$ then $\mathrm{TV} \equiv 0$ and $S(p)=D(p)=0, \forall p$ (see Figure 1 ).

Remark 15. Since we assume the distribution $\rho$ to be absolutely continuous with respect to the Lebesgue measure, it holds that $\operatorname{diam}(\operatorname{supp}(\rho))>0$. Thus one can always find a critical value $\lambda^{*}$ defined as

$$
\lambda^{*}= \begin{cases}\frac{\operatorname{diam}(\operatorname{supp}(\rho))^{2}}{8\left(\underline{\left.f^{\prime}(0)\right)_{+}}\right.} & \text {if }\left(\underline{\left.f^{\prime}(0)\right)_{+}}>0,\right. \\ 0 & \text { if }\left(\underline{\left.f^{\prime}(0)\right)_{+}}=0,\right.\end{cases}
$$

such that for any $\lambda<\lambda^{*}$, the assumptions of Theorem 13 are satisfied; that is, there exists a market price maximizing the volume and clearing the market. On the contrary there exists no such market price for $\lambda \geq \lambda^{*}$. The results of [19] are a special case of this remark. In fact, under the assumptions given in [19], $\left(\underline{\left.f^{\prime}(0)\right)}+{ }_{+}=f^{\prime}(0)=0\right.$ and thus $\lambda^{*}=0$.

The critical value $\lambda^{*}$ can be interpreted as the maximum risk aversion allowing the market to function. If the risk aversion becomes larger than the critical value, the market stops and a liquidity crisis occurs. In the latter case, several actions can be proposed to stop the liquidity crisis:

(i) lowering the perception of risk, that is, lower the $\lambda$ of the agents;

(ii) making $\lambda^{*}$ higher by lowering $\left(f^{\prime}(0)\right)$, that is, lower the marginal cost of information around zero precision. In other words, eliminate any entry barriers for new agents on that market by largely spreading information about the real situation of the asset $V$;

(iii) making $\lambda^{*}$ higher by increasing $\operatorname{diam}(\operatorname{supp}(\rho))$. This means inviting to the market agents with new, different evaluation procedures. This can be carried out for instance by eliminating any entry barrier for newcomers when they have a different background and different evaluation procedures.

3.1. Necessary and Sufficient Results for General Functions. In this section we relax the assumption $\left(f^{\prime}(0)\right)+\infty$. For any function $h$ we denote by $h^{*}$ the Legendre-Fenchel transform (cf. [27]) of $h$ and by $h^{* *}$ the Legendre-Fenchel transform applied twice, and so on. We show in this section that the twice Legendre-Fenchel transform $f^{* *}$ of the cost function $f$ has remarkable properties; that is, we can replace $f$ by $f^{* *}$ for any practical means. In particular this means that from a technical point of view one can suppose that $f$ is convex even if the actual function is not.

Theorem 16. Let $f$ be a function satisfying Assumption 3. The following properties hold for $f^{* *}$ :

(1) $f^{* *}$ also satisfies Assumption 3;

(2) except for a countable set of values $\alpha(p, A)$, one has

$$
\begin{aligned}
& B_{o p t}(p, A ; f)=B_{o p t}\left(p, A ; f^{* *}\right), \\
& \theta_{o p t}(p, A ; f)=\theta_{o p t}\left(p, A ; f^{* *}\right) ;
\end{aligned}
$$

(3) as a consequence

$$
S(f, p)=S\left(f^{* *}, p\right), \quad D(f, p)=D\left(f^{* *}, p\right), \quad \forall p \geq 0 .
$$

Proof. To prove point (1) we recall that $f^{* *}$ is a convex function and $\forall b \geq 0, f^{* *}(b) \leq f(b)$. In particular $f^{* *}$ is l.s.c. and continuous in 0 . Let us now check the growth condition and take $\beta$ that satisfies Assumption 3 for $f$. Take also $C_{1}$ as the constant in Lemma 5; that is, $f(x) \geq C_{1} x^{1+\beta}$ for all $x \geq x_{1}$. Consider now the function

$$
f_{1}(x)=\left\{\begin{array}{ll}
0 & \text { if } x \leq x_{1} \\
C_{1} x^{1+\beta} & \text { if } x>x_{1}
\end{array} .\right.
$$

Then it is straightforward to see that

$$
f_{1}^{* *}(x)= \begin{cases}0 & \text { if } x \leq x_{1} \\ C_{1}(1+\beta) x_{2}^{\beta}\left(x-x_{1}\right) & \text { if } x_{1} \leq x \leq x_{2} \\ C_{1} x^{1+\beta} & \text { if } x \geq x_{2}\end{cases}
$$

where $x_{2}=((1+\beta) / \beta) x_{1}$; of course $f_{1} \leq f$ and is l.s.c. Then we also have the inequality $f_{1}^{* *} \leq f^{* *}$. But obviously $\liminf \operatorname{in}_{x \rightarrow \infty} f_{1}^{* *}(x) / x^{1+\beta}=C_{1}>0$. Hence $\liminf _{x \rightarrow \infty} f^{* *}(x) / x^{1+\beta}>0$.

To prove point (2) we recall that the cost function $f$ is used only as a part of the function $g_{\alpha}$. Let us take a point $\alpha_{0}$ and $x_{0}$ a minimum of $g_{\alpha_{0}}$. This implies

$$
\alpha_{0} x_{0}-f\left(x_{0}\right) \geq \alpha_{0} x-f(x) \quad \forall x
$$


which can also be written as

$$
f(x) \geq f\left(x_{0}\right)+\alpha_{0}\left(x-x_{0}\right) .
$$

That is, as stated in [27], the function $f$ has a supporting hyperplane at $x_{0}$. Since $f$ has a supporting hyperplane at $x_{0}$ this implies that $f\left(x_{0}\right)=f^{* *}\left(x_{0}\right)$; recall that $f^{* *}$ is the convex hull of $f$, that is, the largest convex function such that $f^{* *} \leq f$. Hence, recall that for any function $f^{* * *}=f^{*}$,

$$
\begin{aligned}
\alpha_{0} x_{0}-f^{* *}\left(x_{0}\right) & =\alpha_{0} x_{0}-f\left(x_{0}\right)=f^{*}\left(\alpha_{0}\right) \\
& =f^{* * *}\left(\alpha_{0}\right)=\max _{x} \alpha_{0} x-f^{* *}(x)
\end{aligned}
$$

We thus obtained that $x_{0}$ is a maximum of $\alpha_{0} x-f^{* *}(x)$.

Therefore, if $f$ is replaced by $f^{* *}$, the minimization problem involving $g_{\alpha}$ gives the same solution, except possibly a countable set of values $\alpha$ where the maximum is attained (either for $f$ or $f^{* *}$ ) in more than one point.

Point (3) is a mere consequence of point (2).

For all purposes of calculating aggregate supply and demand we can thus replace $f$ by $f^{* *}$, that is, replace $f$ by its convex hull. Therefore, one can work as if $f$ was convex.

Remark 17. This result is particularly useful when $f(0) \neq f(0)$ because in this situation $\left(f^{\prime}(0)\right)=\infty$. Then one cannot use the previous results that guarantee the uniqueness of the market clearing price. When $f$ is replaced by $f^{* *}$ it can be shown that $\left(f^{\prime}(0)\right)$ becomes finite and the results apply for $f^{* *}$. However Theorem 16 allows recovering the results for the initial function $f$ and obtaining the full information on the supply and demand functions and on the market price.

We obtain the following.

Theorem 18. Suppose that Assumptions 3 and 9 are satisfied. Then there exists at least one price $\overline{\mathscr{P}} \geq 0$ such that

$$
T V(\overline{\mathscr{P}}) \geq T V(p), \quad \forall p \geq 0 .
$$

This price also satisfies

$$
D(\overline{\mathscr{P}})=S(\overline{\mathscr{P}})
$$

\section{Furthermore, consider the following.}

(I) If there exists $B>0$ such that $f(B)<f(0)$, then $D(p ; f)$ and $S(p ; f)$ are always strictly positive and strictly monotonic, $S(0)=0=\lim _{p \rightarrow \infty} D(p)$. Moreover the price $\overline{\mathscr{P}}$ satisfying (35) is unique.

(II) Suppose now that $f(B) \geq f(0), \forall B \geq 0$; then the following hold:

(a) (alternative 1) suppose that $\operatorname{diam}(\operatorname{supp}(\rho))>$ $2 \sqrt{2 \lambda\left(\left(f^{* *}\right)^{\prime}(0)\right)}$; then

(i) the functions $B_{o p t}$ and $\theta_{o p t}$ are well defined, (ii) the price $\overline{\mathscr{P}}$ satisfying (35) is unique and $T V(\overline{\mathscr{P}})>0 ; \overline{\mathscr{P}}$ is also the unique solution of (36);

(b) (alternative 2) if on the contrary one supposes that

$$
\operatorname{diam}(\operatorname{supp}(\rho)) \leq 2 \sqrt{2 \lambda\left(\underline{\left.\left(f^{* *}\right)^{\prime}(0)\right)_{+}},\right.}
$$$$
\text { then } T V(p)=0, \forall p \geq 0 \text {. }
$$

Proof. We prove first point (I). If $f\left(B^{*}\right)<f(0)$, then for all $\alpha \geq 0, \alpha B^{*}-f\left(B^{*}\right)>\alpha \cdot 0-f(0)$; thus $B_{\text {opt }}(p, A)>0$ for all $p, A$. As a consequence we obtain $D(p ; f)>0$ for all $p$ and the same for $S(p ; f)$. For strict monotonicity it suffices to use same arguments as in the proof of Lemma 11. Of course, $S(0)=0=\lim _{p \rightarrow \infty} D(p)$ due to Lemma 10.

The point (IIa) follows from the discussion above.

To prove (IIb) we need to analyze in greater detail the values of $D(p)$ and $S(p)$. If we consider $B_{\text {opt }}\left(p, A ; f^{* *}\right)>0$, then $\alpha B_{\text {opt }}\left(p, A ; f^{* *}\right)-f^{* *}\left(B_{\text {opt }}\left(p, A ; f^{* *}\right)\right)>\alpha \cdot 0-f^{* *}(0)$ (we exclude the null measure set of $\alpha$ where more than one maximum can exists; that is, we can suppose the inequality to be strict); hence

$$
f^{* *}\left(B_{\mathrm{opt}}\left(p, A ; f^{* *}\right)\right)<f^{* *}(0)+\alpha B_{\mathrm{opt}}\left(p, A ; f^{* *}\right),
$$

or, for some $\alpha_{1}<\alpha$,

$$
f^{* *}\left(B_{\mathrm{opt}}\left(p, A ; f^{* *}\right)\right) \leq f^{* *}(0)+\alpha_{1} B_{\mathrm{opt}}\left(p, A ; f^{* *}\right) .
$$

Since $f^{* *}$ is convex we have for arbitrary $B \in$ $\left[0, B_{\text {opt }}\left(p, A ; f^{* *}\right)\right], f^{* *}(B) \leq f^{* *}(0)+\alpha_{1} B$. But this means

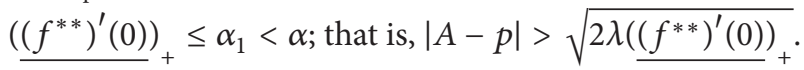

If $D(p)$ is always zero, the conclusion is reached. Suppose now $p$ exists such that $D(p)>0$; then at least some $A$ in the support of $\rho$ exists such that $B_{\text {opt }}\left(p, A ; f^{* *}\right)>0$ and $(A-p)_{+}>0$; the three conditions imply

$$
\sup (\operatorname{supp}(\rho))-\sqrt{2 \lambda\left(\underline{\left.\left(f^{* *}\right)^{\prime}(0)\right)_{+}}\right.}>0 .
$$

Moreover, we have $D(p)=0$ for $p \geq \sup (\operatorname{supp}(\rho))-$ $\sqrt{\left.2 \lambda\left(f^{* *}\right)^{\prime}(0)\right)}$.

From (40) and (37), we conclude that

$$
\begin{aligned}
0 & <\sup (\operatorname{supp}(\rho))-\sqrt{2 \lambda\left(\underline{\left.\left(f^{* *}\right)^{\prime}(0)\right)_{+}}\right.} \\
& \leq \sqrt{2 \lambda\left(\underline{\left.\left(f^{* *}\right)^{\prime}(0)\right)_{+}}+\inf (\operatorname{supp}(\rho)) .\right.}
\end{aligned}
$$

A similar reasoning as the above shows that $S(p)=0$ for $p \leq$ $\sqrt{2 \lambda\left(\left(^{* *}\right)^{\prime}(0)\right)}+\inf (\operatorname{supp}(\rho))$. Therefore for any $p$ either $D(p)=0$ or $S(p)=0$ and the conclusion follows.

In general, the price $\overline{\mathscr{P}}$ has an implicit dependence on the cost function $f(\cdot)$ with no particular properties. But when the distribution $\rho$ is completely symmetric around some particular value $p^{1}$; we obtain the following result. 
Theorem 19. Suppose that Assumptions 3 and 9 are satisfied and there exists $p^{1}>0$ such that

$$
\forall y \in \mathbb{R}: \rho\left(p^{1}-y\right)=\rho\left(p^{1}+y\right),
$$

(with the convention that $\rho$ is null on $\mathbb{R}_{-}$); then one can take in Theorem $18 \overline{\mathscr{P}}=p^{1}$.

Proof. The proof follows from the remark that, except possibly for a null measure set of values $\alpha(p, A)$, the function $B_{\text {opt }}(p, A ; f)$ is symmetric around $p$; that is, $B_{\text {opt }}(p, A ; f)=$ $B_{\text {opt }}(p, 2 p-A ; f)$; thus $\theta_{\text {opt }}(p, A ; f)$ is antisymmetric. Since the distribution $\rho$ is symmetric then $D\left(p^{1}\right)=S\left(p^{1}\right)$.

\section{An Application: The Grossman-Stiglitz Framework}

We follow [9] to analyze a classical situation where costly information can be used to lower the uncertainty of the estimation. Note however that in the cited work the equilibrium is reached without modeling the variations in supply and in the absence of the distribution $\rho(A)$.

In the Grossman-Stiglitz model agents can either pay nothing and have a precision $B_{1}$ or pay a fixed $\operatorname{cost} c_{b}$ to gain precision up to level $B_{2}>B_{1}$. This leads to the function

$$
f(B)= \begin{cases}0 & \text { if } B \leq B_{1} \\ c_{b} & \text { if } B_{1}<B \leq B_{2} \\ +\infty & \text { if } B>B_{2} .\end{cases}
$$

The function $f$ does not satisfy assumption in [19] and as such the result therein cannot be used. It however satisfies the Assumption 3; thus using the Theorem 18 we can replace $f$ with the following convex function $f_{\mathrm{GS}}=f^{* *}$ defined as

$$
f_{\mathrm{GS}}(B)= \begin{cases}0 & \text { if } B \leq B_{1} \\ c_{b} \frac{B-B_{1}}{B_{2}-B_{1}} & \text { if } B_{1} \leq B \leq B_{2} \\ +\infty & \text { if } B>B_{2} .\end{cases}
$$

Note that $f_{\mathrm{GS}}$ fulfills Assumption 3 with an arbitrary $\beta \geq 0$. Suppose that the distribution $\rho(A)$ fulfills the requirements in Assumption 9; absolute continuity with respect to Lebesgue measure and a moment of order $1+\epsilon$ (with arbitrary small $\epsilon$ ) has to exist. Then a (equilibrium) market price exists and is unique. Note that $f_{\mathrm{GS}}^{\prime}(0)=0$; thus $\lambda_{\mathrm{GS}}^{*}=0$.

The unsigned demand is

$$
\theta_{\text {opt }}(p, A)= \begin{cases}\frac{(A-p) B_{1}}{\lambda} & \text { if }|A-p|<\frac{2 \lambda c_{b}}{\left(B_{2}-B_{1}\right)} \\ \frac{(A-p) B_{2}}{\lambda} & \text { if }|A-p| \geq \frac{2 \lambda c_{b}}{\left(B_{2}-B_{1}\right)} .\end{cases}
$$

The optimal precision is either $B_{1}$ (first case of (45)) or $B_{2}$ (second case).

\section{Transaction Volume and Marginal Costs}

We describe in the following the relationship between the cost function $f$ and the trading volume.

Theorem 20. Suppose that $f_{1}$ and $f_{2}$ both satisfy Assumption 3 and that $\rho$ satisfies Assumption 9.

(A) Assume that

$$
\frac{f_{2}(y)-f_{2}(x)}{y-x} \geq \frac{f_{1}(y)-f_{1}(x)}{y-x}, \quad \forall x, y \geq 0, x \neq y .
$$

Then $T V_{f_{1}} \geq T V_{f_{2}}$.

(B) In particular if $f_{1}$ and $f_{2}$ are such that

$$
f_{1}^{\prime}\left(X^{+}\right) \leq f_{2}^{\prime}\left(X^{+}\right), \quad f_{1}^{\prime}\left(X^{-}\right) \leq f_{2}^{\prime}\left(X^{-}\right), \quad \forall X \geq 0,
$$

$$
\text { (all are lateral derivatives) then } T V_{f_{1}} \geq T V_{f_{2}} \text {. }
$$

Remark 21. Note that if $f_{1}$ and $f_{2}$ are convex, both lateral derivatives are defined at each point and (A) implies (B); thus for practical purposes (cf. also Section 3.1) the point (B) is not weaker than point $(\mathrm{A})$.

Remark 22. If $f_{1}^{\prime}(X)$ and $f_{2}^{\prime}(X)$ exist at a certain point $X$, then (47) implies that $f_{1}^{\prime}(X) \leq f_{2}^{\prime}(X)$. Thus, the above result is a generalization of the analogous theorem in [19].

Proof. (A) We first show that, except for a countable set of values $\alpha(p, A)$, we have $B_{\text {opt }}\left(p, A ; f_{1}\right) \geq B_{\text {opt }}\left(p, A ; f_{2}\right)$. Fix $p, A$ and denote $B_{k}=B_{o p t}\left(p, A ; f_{k}\right)$ for $k=1,2$. Suppose, by contradiction, that $B_{1}<B_{2}$; recall that, since $B_{1}$ is optimal,

$$
\alpha B_{1}-f_{1}\left(B_{1}\right)>\alpha B_{2}-f_{1}\left(B_{2}\right) .
$$

Thus

$$
\frac{f_{1}\left(B_{2}\right)-f_{1}\left(B_{1}\right)}{B_{2}-B_{1}}>\alpha .
$$

Note that we wrote strict inequality in (48) because we exclude the countable set of values $\alpha(p, A)$ where the maximum of $g_{p, A}(B)=\alpha B-f_{1}(B)$ is not unique. We do the same for $B_{2}$ :

$$
\alpha B_{2}-f_{2}\left(B_{2}\right)>\alpha B_{1}-f_{2}\left(B_{1}\right) .
$$

Thus

$$
\alpha>\frac{f_{2}\left(B_{2}\right)-f_{2}\left(B_{1}\right)}{B_{2}-B_{1}} .
$$

Combining (49) and (51) we obtain

$$
\frac{f_{1}\left(B_{2}\right)-f_{1}\left(B_{1}\right)}{B_{2}-B_{1}}>\frac{f_{2}\left(B_{2}\right)-f_{2}\left(B_{1}\right)}{B_{2}-B_{1}} .
$$

This, however, contradicts (46) for $y=B_{2}$ and $x=B_{1}$. Thus, with the possible exception of a countable set of values $\alpha(p, A)$, we have $B_{\text {opt }}\left(p, A ; f_{1}\right) \geq B_{\text {opt }}\left(p, A ; f_{2}\right)$. 
The demand and supply of the agents are monotonic and given for $k=1,2$ by the formulas

$$
\begin{gathered}
D\left(f_{k}, p\right)=\frac{1}{2 \lambda} \int_{0}^{\infty}(A-p)_{+} B_{\text {opt }}\left(p, A ; f_{k}\right) \rho(A) d A, \\
S\left(f_{k}, p\right)=\frac{1}{2 \lambda} \int_{0}^{\infty}(A-p)_{-} B_{\text {opt }}\left(p, A ; f_{k}\right) \rho(A) d A .
\end{gathered}
$$

Let $P_{f_{k}}^{A}$ be the market price for which supply equals demand for the cost function $f_{k}$; that is, $D\left(f_{k}, P_{f_{k}}^{A}\right)=$ $S\left(f_{k}, P_{f_{k}}^{A}\right)$. We further take $P_{f_{2}}^{A}=\min \left\{P: D\left(f_{2}, P\right)=S\left(f_{2}, P\right)\right\}$ and $P_{f_{1}}^{A}=\min \left\{P: D\left(f_{1}, P\right)=S\left(f_{1}, P\right)\right\}$.

It has been proved that $B_{\text {opt }}\left(p, A ; f_{1}\right) \geq B_{\text {opt }}\left(p, A ; f_{2}\right)$. Thus, $D\left(f_{1}, p\right) \geq D\left(f_{2}, p\right)$ and $S\left(f_{1}, p\right) \geq S\left(f_{2}, p\right), \forall p$. In particular, $D\left(f_{2}, P_{f_{2}}^{A}\right) \leq D\left(f_{1}, P_{f_{2}}^{A}\right)$.

Let $P_{1}$ be the solution of $D\left(f_{1}, P_{1}\right)=S\left(f_{2}, P_{1}\right)$. Let us prove that $P_{1} \geq P_{f_{2}}^{A}$. Suppose, on the contrary, that $P_{1}<P_{f_{2}}^{A}$. Then

$$
\begin{aligned}
D\left(f_{1}, P_{f_{2}}^{A}\right) & \geq D\left(f_{2}, P_{f_{2}}^{A}\right)=S\left(f_{2}, P_{f_{2}}^{A}\right) \\
& \geq S\left(f_{2}, P_{1}\right)=D\left(f_{1}, P_{1}\right) \geq D\left(f_{1}, P_{f_{2}}^{A}\right),
\end{aligned}
$$

which means that all inequalities in (54) are in fact equalities, in particular $S\left(f_{2}, P_{f_{2}}^{A}\right)=S\left(f_{2}, P_{1}\right)$ and $D\left(f_{1}, P_{1}\right)=D\left(f_{2}, P_{f_{2}}^{A}\right)$. But we also have

$$
D\left(f_{1}, P_{1}\right) \geq D\left(f_{2}, P_{1}\right) \geq D\left(f_{2}, P_{f_{2}}^{A}\right)=D\left(f_{1}, P_{1}\right)
$$

which means again that all terms are equal, in particular $D\left(f_{2}, P_{1}\right)=D\left(f_{2}, P_{f_{2}}^{A}\right)$. Thus

$$
D\left(f_{2}, P_{1}\right)=D\left(f_{2}, P_{f_{2}}^{A}\right)=S\left(f_{2}, P_{f_{2}}^{A}\right)=S\left(f_{2}, P_{1}\right),
$$

which means that $P_{1}$ is a member of $\left\{P: D\left(f_{2}, P\right)=S\left(f_{2}, P\right)\right\}$. However as $P_{f_{2}}^{A}$ is the minimum of such elements we arrive at a contradiction. It follows that $P_{1} \geq P_{f_{2}}^{A}$.

Similarly we prove that $P_{1} \geq P_{f_{1}}^{A}$ (see Figure 2 ). Hence it holds that

$$
\begin{aligned}
\mathrm{TV}_{f_{2}} & =S\left(f_{2}, P_{f_{2}}^{A}\right) \leq S\left(f_{2}, P_{1}\right)=D\left(f_{1}, P_{1}\right) \\
& \leq D\left(f_{1}, P_{f_{1}}^{A}\right)=\mathrm{TV}_{f_{1}},
\end{aligned}
$$

which concludes the proof.

(B) We prove that (47) implies (46). Of course, it is enough to consider $x<y$. Denote

$$
\begin{array}{r}
G(y, x)=\frac{f_{2}(y)-f_{2}(x)}{y-x}-\frac{f_{1}(y)-f_{1}(x)}{y-x}, \\
\forall x, y \geq 0, \quad x \neq y .
\end{array}
$$

Suppose that $x_{0}$ and $y_{0}>x_{0}$ exist such that $\xi:=G\left(y_{0}\right.$, $\left.x_{0}\right)<0$. Note that

$$
G(y, x)=\frac{1}{2} G\left(y, \frac{x+y}{2}\right)+\frac{1}{2} G\left(\frac{x+y}{2}, x\right) .
$$

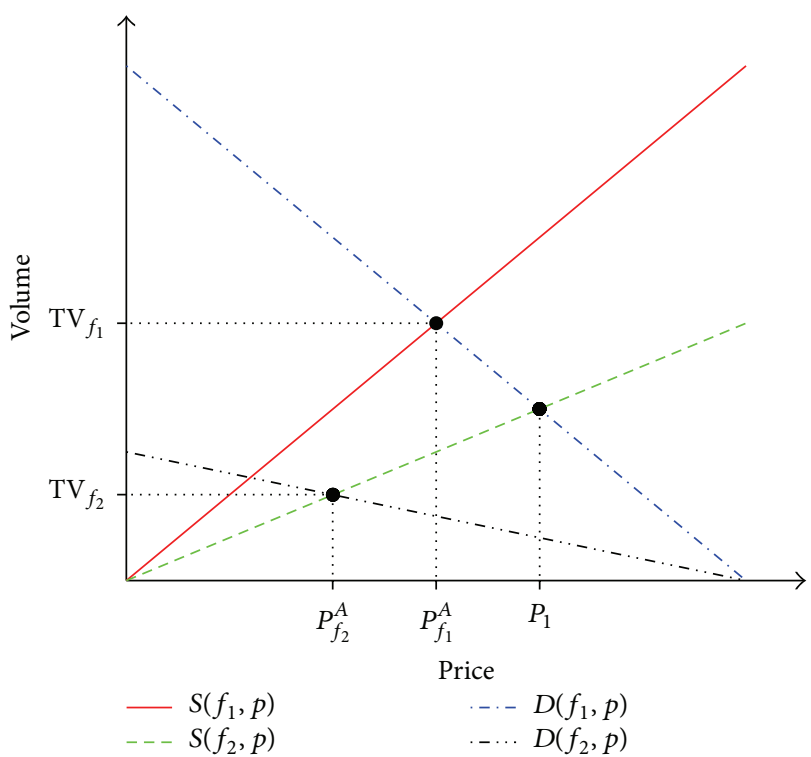

FIGURE 2: Illustration of the proof of Theorem 20.

Then, either $G\left(y_{0},\left(x_{0}+y_{0}\right) / 2\right) \leq \xi<0$ or $G\left(\left(x_{0}+y_{0}\right) / 2, x_{0}\right) \leq$ $\xi<0$. Iterating the argument we obtain two convergent sequences $x_{n}$ and $y_{n}$ with $\lim _{n \rightarrow+\infty} y_{n}=\lim _{n \rightarrow+\infty} x_{n}=$ $x_{\infty}, x_{n}<y_{n}$, and $G\left(y_{n}, x_{n}\right) \leq \xi<0$. Up to extracting subsequences only three alternatives exist:

(1) $x_{\infty} \leq x_{n}<y_{n}$ for all $n$,

(2) $x_{n}<y_{n} \leq x_{\infty}$ for all $n$,

(3) $x_{n} \leq x_{\infty} \leq y_{n}$ for all $n$.

Alternative (3) can be reduced to (1) or (2) by noting that since $G\left(y_{n}, x_{n}\right)=\left(\left(y_{n}-x_{\infty}\right) /\left(y_{n}-x_{n}\right)\right) G\left(y_{n}, x_{\infty}\right)+\left(\left(x_{\infty}-\right.\right.$ $\left.\left.x_{n}\right) /\left(y_{n}-x_{n}\right)\right) G\left(x_{\infty}, x_{n}\right)$ then either $G\left(y_{n}, x_{\infty}\right) \leq \xi$ or $G\left(x_{\infty}, x_{n}\right) \leq \xi<0$.

We only prove (1), the proof of (2) being completely similar. When $x_{\infty} \leq x_{n}<y_{n}$ we obtain

$$
0>\xi \geq \lim _{n \rightarrow+\infty} G\left(y_{n}, x_{n}\right)=f_{2}^{\prime}\left(x_{\infty}^{+}\right)-f_{1}^{\prime}\left(x_{\infty}^{+}\right) \geq 0,
$$

which is a contradiction. Thus (47) implies (46).

\section{Concluding Remarks}

The main focus of this work is to establish the existence of an equilibrium and its optimality in terms of trading volumes for the model in Section 2. The results are proved under minimal assumptions on the cost function and a relationship with the convex hull of the cost function is proved. The model can be used to investigate the determinants of the trading volume and may give hints on how to exit a situation when the volume is abnormally low.

\section{Conflict of Interests}

The authors declare that there is no conflict of interests regarding the publication of this paper. 


\section{References}

[1] J. M. Keynes, The General Theory of Employment, Interest and Money, Macmillan, London, UK, 1936.

[2] H. M. Wu and W. C. Guo, "Speculative trading with rational beliefs and endogenous uncertainty," Economic Theory, vol. 21, no. 2-3, pp. 263-292, 2003.

[3] H. M. Wu and W. C. Guo, "Asset price volatility and trading volume with rational beliefs," Economic Theory, vol. 23, no. 4, pp. 795-829, 2004.

[4] J. A. Scheinkman and W. Xiong, "Heterogeneous beliefs, speculation and trading in financial markets," in Paris-Princeton Lectures on Mathematical Finance 2003, R. A. Carmona, E. Cinlar, I. Ekeland, E. Jouini, J. A. Scheinkman, and N. Touzi, Eds., vol. 1847 of Lecture Notes in Mathematics, pp. 217-250, Springer, Berlin, Germany, 2004.

[5] H. R. Varian, "Divergence of opinion in complete markets: a note," The Journal of Finance, vol. 40, no. 1, pp. 309-317, 1985.

[6] M. Harris and A. Raviv, "Differences of opinion make a horse race," The Review of Financial Studies, vol. 6, no. 3, pp. 473-506, 1993.

[7] S. Morris, "Speculative investor behavior and learning," Quarterly Journal of Economics, vol. 111, no. 4, pp. 1111-1133, 1996.

[8] M. Pagano, "Endogenous market thinness and stock price volatility," The Review of Economic Studies, vol. 56, no. 2, pp. 269-287, 1989.

[9] S. J. Grossman and J. E. Stiglitz, "On the impossibility of informationally efficient markets," The American Economic Review, vol. 70, no. 3, pp. 393-408, 1980.

[10] J. B. de Long, A. Shleifer, L. H. Summers, and R. J. Waldmann, "Noise trader risk in financial markets," Journal of Political Economy, vol. 98, no. 4, pp. 703-738, 1990.

[11] J. Wang, "A model of competitive stock trading volume," Journal of Political Economy, vol. 102, no. 1, pp. 127-168, 1994.

[12] R. E. Verrecchia, "Information acquisition in a noisy rational expectations economy," Econometrica, vol. 50, no. 6, pp. 14151430, 1982.

[13] M. O. Jackson, "Equilibrium, price formation, and the value of private information," The Review of Financial Studies, vol. 4, no. 1, pp. 1-16, 1991.

[14] L. Veldkamp, "Media frenzies in markets for financial information," The American Economic Review, vol. 96, no. 3, pp. 577-601, 2006.

[15] K. J. Ko and Z. (James) Huang, "Arrogance can be a virtue: overconfidence, information acquisition, and market efficiency," Journal of Financial Economics, vol. 84, no. 2, pp. 529-560, 2007.

[16] T. Krebs, "Rational expectations equilibrium and the strategic choice of costly information," Journal of Mathematical Economics, vol. 43, no. 5, pp. 532-548, 2007.

[17] J. Litvinova and H. O. Yang, "Endogenous information acquisition: a revisit of the Grossman-Stiglitz model," Working Paper, Duke University, 2003.

[18] L. Peng, "Learning with information capacity constraints," Journal of Financial and Quantitative Analysis, vol. 40, no. 2, pp. 307-329, 2005.

[19] M. Shen and G. Turinici, "Liquidity generated by heterogeneous beliefs and costly estimations," Networks and Heterogeneous Media, vol. 7, no. 2, pp. 349-361, 2012.

[20] R. S. Bucy and P. D. Joseph, Filtering for Stochastic Processes with Applications to Guidance, Interscience Tracts in Pure and Applied Mathematics, Interscience, New York, NY, USA, 1968.
[21] J. S. Abarbanell, W. N. Lanen, and R. E. Verrecchia, "Analysts' forecasts as proxies for investor beliefs in empirical research," Journal of Accounting and Economics, vol. 20, no. 1, pp. 31-60, 1995.

[22] L. Peng and W. Xiong, "Time to digest and volatility dynamics," Working Paper, Baruch College and Princeton University, 2003.

[23] K. J. Arrow, Aspects of the Theory of Risk-Bearing. Yrjö Jahnsson lectures, Yrjö Jahnssonin Säätiö, Helsinki, Finland, 1965.

[24] S. J. Grossman, "The existence of futures markets, noisy rational expectations and informational externalities," The Review of Economic Studies, vol. 44, no. 3, pp. 431-449, 1977.

[25] S. Grossman, "Further results on the informational efficiency of competitive stock markets," Journal of Economic Theory, vol. 18, no. 1, pp. 81-101, 1978.

[26] S. Grossman, "On the efficiency of competitive stock markets where trades have diverse information," The Journal of Finance, vol. 31, no. 2, pp. 573-585, 1976.

[27] R. T. Rockafellar, Convex Analysis, Princeton University Press, Princeton, NJ, USA, 1970. 


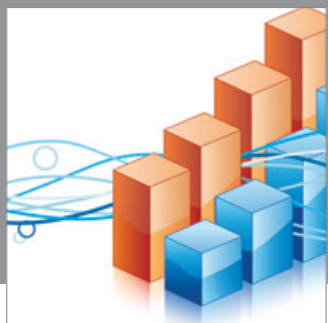

Advances in

Operations Research

mansans

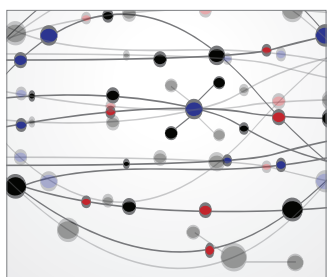

The Scientific World Journal
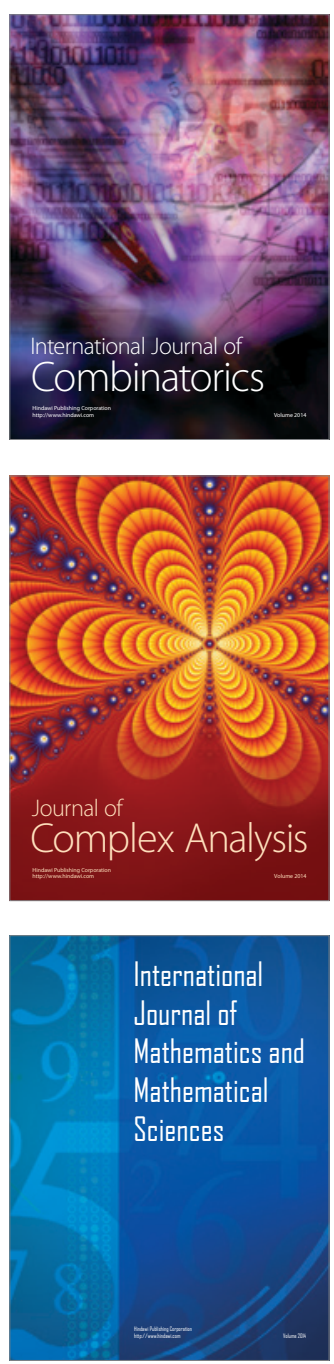
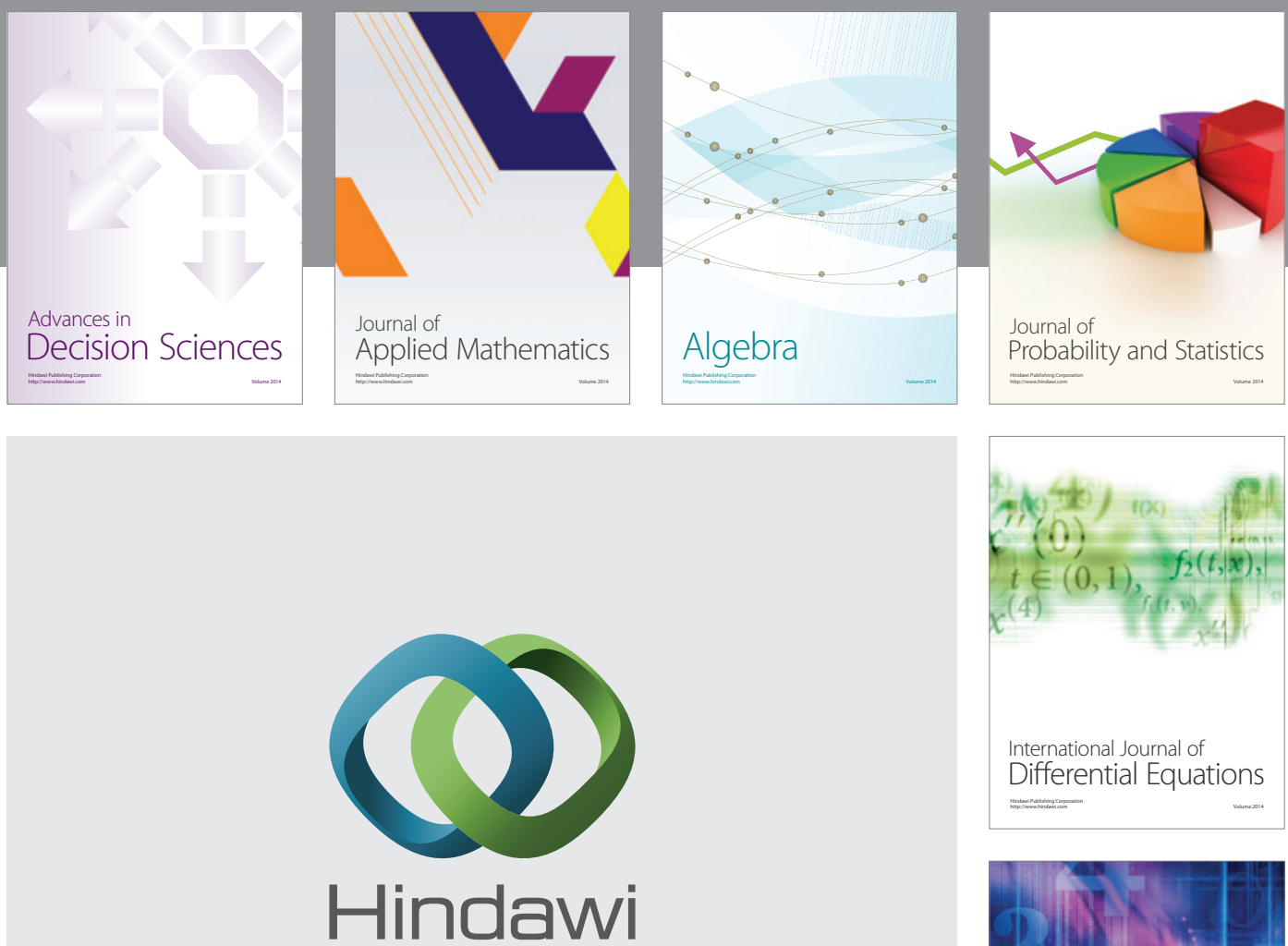

Submit your manuscripts at http://www.hindawi.com
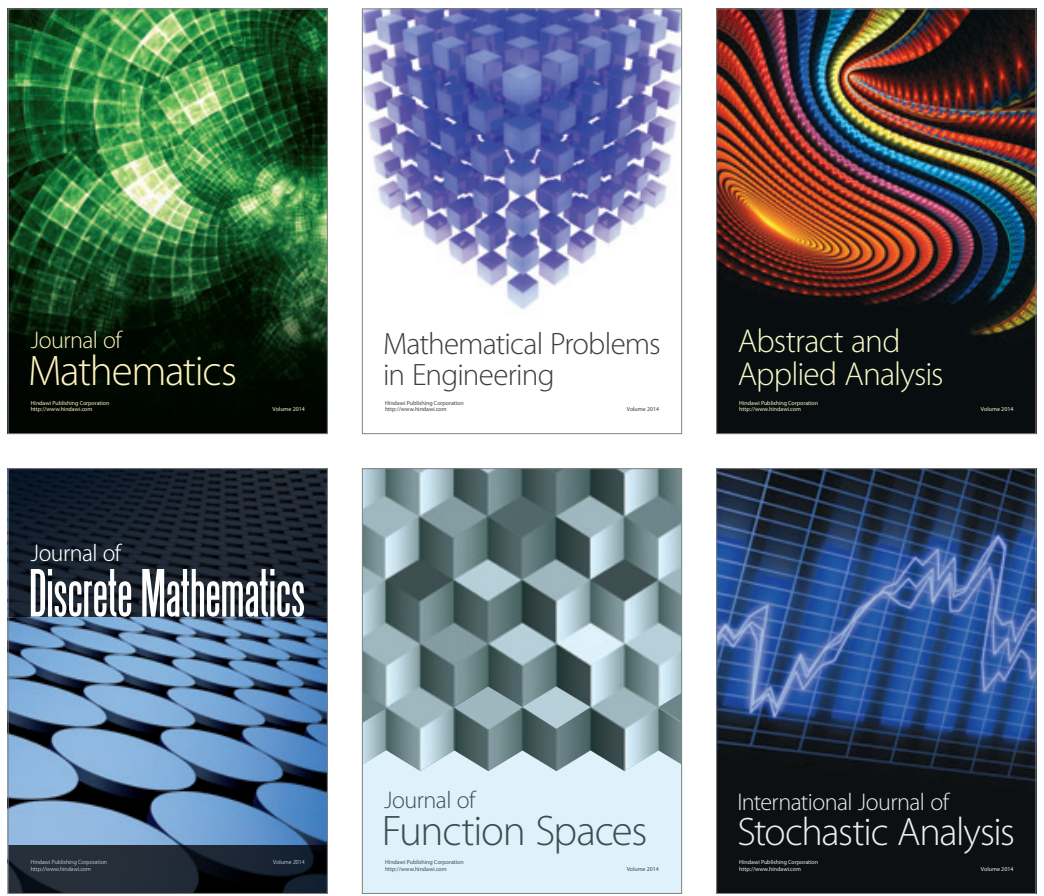

Journal of

Function Spaces

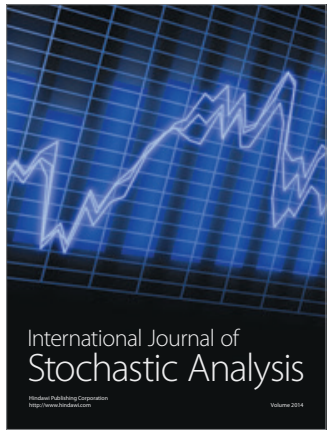

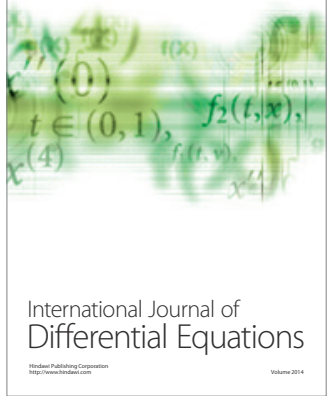
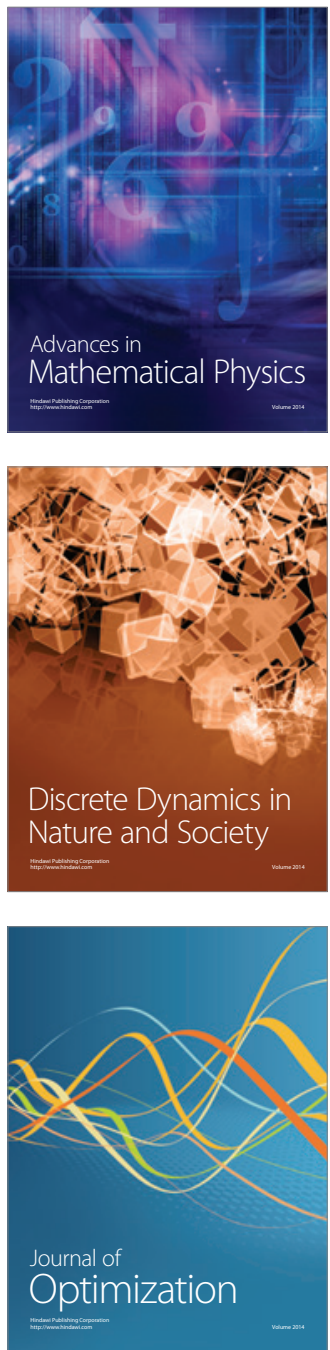